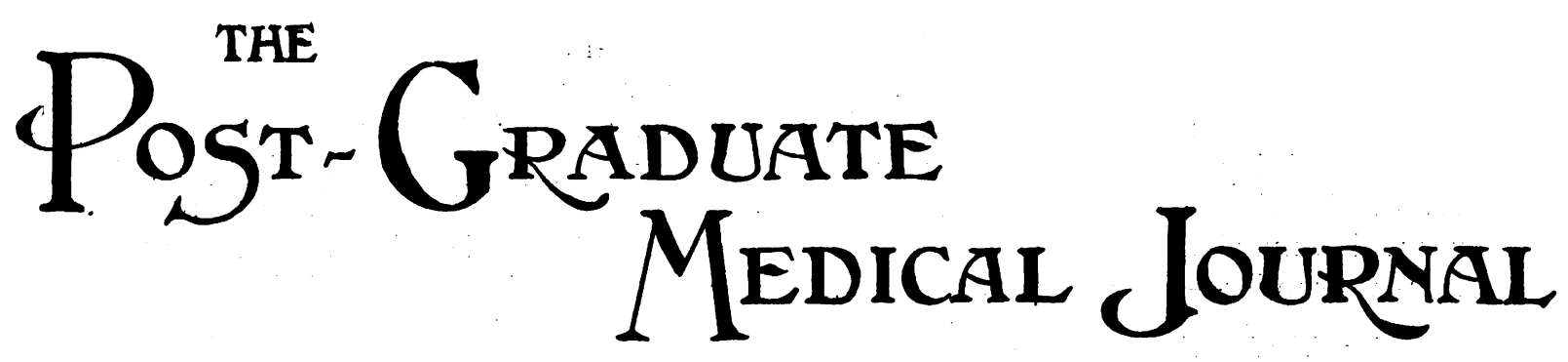

Vol. III. NOVEMBER, 1927. $\quad$ No. 26.

\title{
CONTENTS
}

The Use and Abuse of Antiseptics.

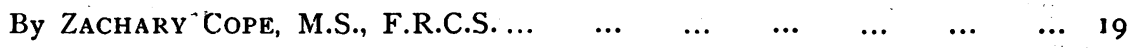

MEdicine AND The STATE.

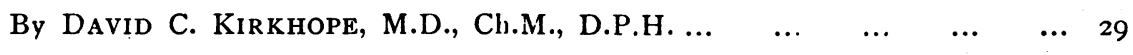

$\begin{array}{llllllllllllll}\text { EDITORIAL NOTES } & \ldots & \ldots & \ldots & \ldots & \ldots & \ldots & \ldots & \ldots & \ldots & \ldots & 33\end{array}$

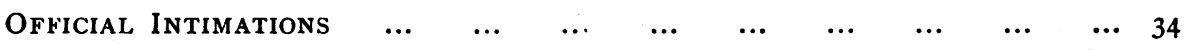

Fellowship of Medicine and Post-Graduate Medical association.-

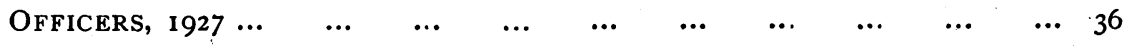

$\begin{array}{lllllllllll}\text { SPECIAL COURSES ... } & \ldots & \ldots & \ldots & \ldots & \ldots & \ldots & \ldots & \ldots & 36\end{array}$

\section{THE USE AND ABUSE OF ANTISEPTICS.}

\author{
$B$ B ZACHARY COPE, \\ M.S., F.R.C.S.,
}

Surgeon to Out-Patients to St. Mary's Hospital, Paddington.

THE oldest and the newest problem in surgery is that of the treatment of wounds. Primitive man applied some healing herb, mediæval chirurgeons poured in wine and oil, whilst modern surgeons have largely trusted to the efficacy of antiseptics to procure the rapid and satisfactory healing of wounds. But during the last decade orthodox views about antiseptics have been rudely shaken, and it is necessary to reconsider the question of their utility.
When first Lister showed that suppuration in wounds could be prevented if the access of microbes rere rendered impossible by the use of antiseptic dressings, there ensued for a time some confusion of thought as to which parts of the antiseptic technique were essential for success. Lister himself was constantly experimenting, and, on the basis of his experiments, discarding unnecessary factors and altering his methods. Pure carbolic was superseded by 1 in 20 solution of the acid, the cart5olic spray was banished, carbolic acid for a time gave way to perchloride of mercury, then zinc chloride was for a time much in favour, and finally double cyanide of mercury and zinc was the drug of choice. In the dressing itself the putty and block-tin gave way to gauze and protective. W.ith all the differences 
of technique Lister had a firm grasp of principles which sometimes eluded his successors. For many years there existedand perhaps still exists-a rivalry between the aseptic and the antiseptic schools. The aseptic school lays stress on the natural power of the tissues to resist infection-a power which may be injured by the application of antiseptics-and asserts that all that is necessary to procure healing is to prevent the access of microbes to the wound. The antiseptic school contend that, in the strength used, antiseptics do very little harm to the tissues and a great deal of good to the patient by the destruction of organisms present. Lister belonged to neither school, for he saw the good in both. His aim was to produce an aseptic wound by antisepsis, but he quite realized that where infection was minimal, aseptic technique could produce as good results as the antiseptic method. He was one of the first, if not the very first, to lay stress on the natural resistance of the body (a resistance much overlooked by many of his pupils), and taught that whenever the body was showing an adequate resistance, it should not be injured by strong antiseptics. Let me quote from his writings some passages which express these views :-

"These two great truths then have been taught us by advancing science; that normal serum is not a good soil for the development of attenuated microbes, and that bacteria introduced among the tissues, if in not too concentrated a form, are disposed of by phagocytosis. The result is that microbes in the form in which they are present in the air are unable to develop in our wounds; and thus we are able to disregard in our operations the once dreaded atmospheric dust."

Again, with reference to the pathology of an acute abscess, we read :-

"I conceive that the acrid products of putrefaction act injuriously upon the pyogenic membrane and prevent des- truction of the micrococci by the natural antisepsis which is always dis posed to operate, but so long as the abscess is unopened, is hindered by the disturbing influence of tension causes by the rapidly accumulating pus." $\frac{\widehat{ }}{\mathrm{Q}}$ Again, in relating the treatment of am acute abscess by incision and drainage, the extremely significant remark is made :-

"The antiseptic substance was nev $\vec{g}$

from first to last applied to the cavity of the abscess, as such treatment coufi only have been productive of needles irritation."

Moreover, even in thuse cases whic $\overrightarrow{\mathrm{A}}$ Lister considered suitable for antiseptic treatment great care was taken, as the following passage shows :-

"In treating surgical cases antise $\frac{\Phi}{2}$ tically, I always endeavoured to avo ind the direct action of the antiseptic spebs stance upon the tissues, so far as consistent in the existing state of knôें ledge with attaining the essential objeest of preventing the development of injurious microbes in the par幙 concerned."

Those who care to read Lister's writing will find also that he was fully aware of the fact that antiseptics do not act so well $\frac{\mathrm{H}}{\mathrm{m}}$ serum as in a watery medium, and was under no illusion as to the rapidity with which an antiseptic locally applied loses ifis power by dilution and combination with tissues. What, then, was the final teaching of Lister regarding antiseptics? We thirk we are correctly representing that teachide by enumerating the purposes for which Lord Lister used antiseptics towards the end of his career.

(I) To sterilize instruments and sometimes towels, \&c.

(2) As a protective dressing to preve $\mathrm{t}$ the access of microbes to the wound and $\$$ prevent the multiplication of putrefactive organisms in the discharges of the wound.

(3) To sterilize or render aseptic receât dirty wounds. In reference to the last $\frac{P_{e}}{2}$ 
writes: "Contused wounds into which dirty material of one kind or another has been introduced before they are seen by the surgeon must be purified by the use of powerful antiseptic means, and must for a while discharge freely."

(4) The purification of skin surfaces.

Lister also recognized that gunshot wounds were in a different category, and though he knew that iodoform had little or no influence over the growth of bacteria outside the body, he made the statement :"On the field of battle iodoform is probably the best means at present at our disposal."

I have thought fit to put on record these views of Lister, since-judging, at least, from my own surgical upbringing and that of contemporaries from other schools-there has been too great a tendency to regard antiseptics of far wider application than they actually are. With the extension of the scope of surgical operations, and the multiplication of theatres where aseptic technique could be practised, antiseptics diminished in use, but away from the operating room they were, and still are, commonly used without much discrimination for applying to septic wounds, for washing out infected cavities, and for sterilizing surfaces. Certainly for many years it has been current teaching that wounds with well-established infection are benefited by treatment with antiseptics, though, as may be judged from the quotations from Lister's writings, the originator of the antiseptic method knew the limitations of the treatment.

This was the state of affairs when the Great War broke out. The limitations of antiseptics became then woefully apparent in the special kind of wound made by highvelocity projectiles, which often bore into the tissues a heavy dose of infective bacteria. The utter failure of the ordinary antiseptic treatment to stop or even modify the terrible infections caused a mental awakening on the subject. The various antiseptics were blamed for not doing what their originator never thought they could do. Much experimental work was carried out. Various antiseptic fluids, pastes, and powders were tried, and in the laboratory an attempt was made to provide some other method of combating wound-infections. The brilliant work of Sir Almroth Wright and his colleagues emphasized the natural antiseptic powers of the tissues, and devised methods for assisting and applying those powers; whilst Browning and others sought for a more potent chemical which should have a greater antiseptic power in serum and yet do little harm to the cellular structures of the body. It would take too long to give a full account of these researches, but it is necessary to summarize their experimental results, and to draw those practical conclusions which are the greatest common measure of both the laboratory and the clinical factors in the problem.

We will consider the action of antiseptics on wounds and on skin and mucous surfaces. It is necessary to discriminate between the various types of wound with reference not to shape or depth, but degree of infection. It is helpful to consider wounds as belonging to one of five classes:-

(1) Clean, i.e., no microbes present, or sufficiently few to be negligible.

(2) Superficially contaminated, i.e., a considerable number of microbes present on the surface, but their growth in the wound not advanced.

(3) Deeply infected, i.e., the organisms have already got a hold on the tissues.

(4) Wounds by high-velocity projectiles, e.g., gunshot wounds.

(5) Granulating wounds:

Under the first group come all operationwounds in healthy tissues when the operation has been carried out under the rules of modern aseptic technique; some accidental wounds also come in this group. If it can be made certain that the tissues were injured by a "clean" instrument, and that no sub sequent contamination has occurred, there is general agreement that no necessity arises 
for the application of an antiseptic. A sterile dressing fulfils all requirements.

The difference of opinion begins when we consider a wound to which microbes have gained access. In the superficially contaminated wound the microbes have been deposited on the surface of the wound, but have not penetrated to any appreciable depth into the tissues. Most civil wounds are of this type for some hours after their infliction. The infected wound is one in which the organisms have had time to multiply in the wound and to invade the tissues adjacent to the injured surface. All untreated contaminated wounds progress into this class within a few hours of infliction. The special characteristic of wounds produced by high-velocity projectiles distinguishes them from all other wounds, for any bacterial contamination carried in at such a high velocity is thrust into the tissues along the path of the missile, so that the wound must, from the first, be regarded as a deeplyinfected one. It is from the failure to realize how essentially gunshot wounds differ from most civil wounds that disappointment may arise. The treatment for the one class is very different from that of the other. We have nowhere seen this essential distinction sufficiently insisted upon. The surgeons in France soon found that no matter what antiseptic was stuffed into the wound, infection was not prevented. Experience showed that removal of the already deeply-infected area by surgical excision at the earliest opportunity gave the best chance of minimal infection. From these facts the facile, but we believe fallacious, deduction was made in some quarters that chemical antiseptics can have no useful part in the prophylactic treatment of any recently contaminated wound.

Let us consider first the recently and superficially contaminated wound. There are three ways of obtaining information bearing on the question of their treatment by antiseptics. One can try to reprocluce the conditions in a test-tube, experiment on animals, or observe the facts as shown iff human wounds. The experiments which have been made in a test-tube do not encourage us to use antiseptics, for if arte ficial recesses are made in the tube the chemical does not appear to reach the microbes in the recess (Fleming). But it is unwise to draw conclusions from experit ments of this nature where there are so many variable factors and the conditiong cannot even be said to approximate to the conditions in a flesh wound.

The experiments on animals should caris more conviction, since they have to do with contaminated wounds in living tissues. $\overrightarrow{d d}$ is surprising how few experiments of thrs nature have been carried out. The wor which is most apposite is that of Cagl Browning and R. Gulbransen, who exper⿳亠口冋 mented with guinea-pigs, which are veri susceptible to the diphtheria-bacillus. In 8 a subcutaneous pocket of an animal (1. injected a lethal dose of the organism, and at a certain time after the injection swabbed out the pocket with a solution of acriflavine. The swabbing was done oft varying times after infection and wi different strengths of flavine in the caşe of different animals, and control animats were similarly infected and no antiseptectice applied. In every case the control animats died, but in all the animals whose wound had been treated by acriflavine solution, I Ioo to $I$ in $I, 000$ within two hours of the infection, recovery took place. Browning summarizes as follows :-

"The results of treating recent perimental wounds passing into the subcutaneous tissue which have beên infected with highly virulent diphtheriga bacilli prove the efficacy of antiseptic therapy. Under the conditions inves gated, brief treatment with a suitale antiseptic (neutral acriflavine in coscentration, $I$ in $I 00$ to $I$ in $I, 000$ ) hiags caused survival of practically roo psis cent. of the infected animals. On the other hand, salt solution, either $0.85 \overrightarrow{\mathcal{B}} \mathrm{r}$ 
5 per cent. $\mathrm{NaCl}$, similarly applied, did not prevent in any instance the acute illness and fatal termination."

These experiments on animals are very striking and carry conviction as to the value of acriflavine in that particular class of contaminated wounds. It does not necessarily follow that the same or similar chemicals would have a similar action with regard to the same or other organisms present in human wounds during the initial stage of contamination. This assumption would, however, be justifiable if clinical support were forthcoming, for experiment in vivo, backed up by clinical confirmation, is more to be trusted than mere experiment in vitro.

Now this third evidential factor-that of experience with contaminated human wounds-gives strong support to the results of the animal experiments. Given a recent superficially contaminated wound of an open or accessible type, it is a cominon experience to find that the application of a suitable antiseptic will prevent serious infection, which one knows from knowledge of neglected cases would in the great majority of instances inevitably ensue. (We must insist again that gunshot wounds are not included in this category.) Contaminated compound fractures, lacerated and dirty wounds, will usually heal with little or no signs of bacterial infection, if treated thoroughly with a suitable antiseptic within two hours (or thereabouts) of the infliction of the wound. It was in the recently contaminated wound that Lister first unequivocably demonstrated the value of antiseptics. So long as the microbes on the surface of the wound are destroyed, it does not signify even if a considerable layer of cellular tissue is also disintegrated, for Lister again and again showed that, so long as the initial infection was combated and the access of further organisms prevented, the necrotic tissue underwent a slow process of nonsuppurative absorption.

It was after reading of Browning's experiments that we once more referred to the eleven cases related by Lister in his first communication on the treatment of compound fractures by antiseptics. In all but one of the cases the wound was thoroughly swabbed with pure carbolic acid. Fortunately in all but the first two cases mention is made of the time elapsing between the accident and application of the antiseptic.

\begin{tabular}{|c|c|c|c|c|}
\hline \multirow{5}{*}{$\begin{array}{c}\text { Case } \\
3 \\
4 \\
5\end{array}$} & \multicolumn{3}{|c|}{$\begin{array}{l}\text { Time elaps- } \\
\text { ing between } \\
\text { accident and } \\
\text { treatment. }\end{array}$} & \multirow{2}{*}{ Result. } \\
\hline & & Hour & & \\
\hline & $\ldots$ & $3 \frac{1}{2}$ & $\ldots$ & No suppuration. \\
\hline & $\cdots$ & 2 & $\ldots$ & No suppuration. \\
\hline & .. & 3 & $\ldots$ & $\begin{array}{l}\text { Superficial discharge due to } \\
\text { carbolic irritation. }\end{array}$ \\
\hline 6 & $\ldots$ & 6 & $\ldots$ & $\begin{array}{l}\text { Deep abscess and secondary } \\
\text { hæmorrhage. }\end{array}$ \\
\hline 7 & $\ldots$ & 6 & $\ldots$ & $\begin{array}{l}\text { No suppuration (s mall } \\
\text { wound). }\end{array}$ \\
\hline 8 & $\ldots$ & 4 & $\ldots$ & $\begin{array}{l}\text { Suppuration (carbolic in oil } \\
\text { used). }\end{array}$ \\
\hline 9 & $\ldots$ & $I \frac{1}{2}$ & $\ldots$ & No suppuration. \\
\hline IO & ... & $\mathbf{I}$ & $\ldots$ & No suppuration. \\
\hline & .. & $\mathbf{I}$ & $\ldots$ & No suppuration. \\
\hline
\end{tabular}

In Case 5 hospital gangrene occurred in a small wound (on the same limb) to which no antiseptic had been applied.

Though this series is a small one, it derives great importance from the fact that the details of the wounds are very carefully set down by Lister himself. The confirmation in the case of human wounds of the results obtained by Browning in experiments on animals is so striking that it must impress everyone. Similar results are of course obtained whenever antiseptics are used rationally in recent civil wounds. In applying antiseptics to a recent wound there is no question of trying to prevent injury to leucocytes or other tissues, for the emigration of leucocytes in sufficient numbers to be of appreciable value takes time, and their effect must be neglected in the attempt to attain primary sterilization. This attempt is considerably furthered by excising bruised and crushed tissues and removing gross dirt, into the midst of which the experiments of Fleming show it to be unlikely for any antiseptic to penetrate. 
We conclude that it is sound surgery to treat recent superficially contaminated wounds with an antiseptic. The casualtyroom should clearly be run on antiseptic lines. For reasons which we give later, we think acriflavine, $I$ in $I, 000$, the best solution to use, but good results may be expected from other solutions.

Wounds with Established Infection.-The problem is very different when the microbes have had time to multiply in the wound and to grow out into the contiguous tissues. Here it is not a question of destroying organisms upon the surface, but of reaching them some distance beneath the surface. The natural physiological reaction will already have begun and the leucocytes and serum will be combating the infection, whilst the processes of tissue-repair will be set in action in an attempt to make granulation tissue. The $a$ priori chances of an antiseptic doing good in such a wound are not promising, for substances which injure organisms will be likely to damage the natural processes of repair: It will be of advantage to consider more closely the pathology of a septic wound.

When micro-organisms are planted in a wound their growth and development depend upon several factors. On the one hand the virulence of the microbe and the opportunity it has for growth, on the other hand the protective apparatus of the body-tissues which comprise : ( $I$ ) The antibacterial action of the exuded serum; (2) the phagocytic and bactericidal action of the leucocytes; (3) the formation of a protective layer of granulation tissue. The growth of an organism is favoured by the presence of stagnant blood-serum and disintegrated cell-debris. When leucocytes and other tissue-cells disintegrate a tryptic ferment is liberated. This trypsin helps to digest and get rid of sloughs, but unfortunately the resulting medium is very favourable to the growth of nearly all organisms. As the wound progresses the blood develops an increased antitryptic power which, by means of the exuded serum, takes effect in the wound and helps to get rid of that one particular factor which en courages the growth of germs. It should béquite clear, however, that the most importan means of retarding the growth of an organ? ism in the cavity of a wound is to get rid oo sloughs and drain away the stagnant dis $\frac{n}{\%}$ charges which have no antibacterial content This material which has been termed either putrid (Lister) or corrupt (Wright) must be allowed an exit, so it is universally admittect that appropriate drainage of a septic woun $\frac{\text { क }}{8}$ is essential. This factor of drainage is in $\frac{0}{3}$ deed the ONLY point upon which all clinicati observers and laboratory-workers do agree. $\tilde{\sim}$

Granulation-tissue takes some three or fout days to form, even under favourable cong ditions (and conditions cannot be said to be favourable when organisms are present), soo it is evident that for a few days the natura defensive powers are the serum and the leucocytes. We have already quoted from Lister's writing to show his teaching as the antibacterial influence of serum. T Tis line of investigation has been fruitfu倮 followed up by Wright, who demonstratede that though human serum possessed th@ power to kill many microbes, yet some o믈 the most deadly organisms had the powe $\overrightarrow{5}$ of living and even growing in serum (sero 3 phytic). The streptococcus and the staphylococcus are examples of serophytic organisms. A flow of serum, therefore, into the wound is advantageous in getting rid os aerobic and anaerobic bacilli, but is of little value in combating the dreaded strepto $\frac{O}{3}$ coccus and the annoying and ubiquitous staphylococcus. For defence against these the natural resource is the leucocyte, whiche. works either as a direct killer of septiơ organisms or, in conjunction with the opsonic properties of the serum, as a devourein of the cocci (phagocytosis).

In an infected wound wherein microbeso are deeply entrenched it is important not top interfere with the natural defensive forces; ${ }_{i}^{\circ}$ since the action of the antiseptic on the microbes is dubious and difficult to ascertain $\frac{\vec{\nabla}}{\mathrm{D}}$ To what extent do antiseptics interfere with⿳亠丷厂巾 
the natural defence? For our answer to this question we are deeply indebted to Alexander Fleming, who found that though in the concentrations commonly used for treatment of wounds most antiseptics will inhibit the growth of organisms in serum, and even in some cases kill organisms in pus, yet they at the same time retard the emigration of leucocytes and diminish their phagocytic power.

Concerning the transudation of serum (or at any rate albuminous fluid), Fleming pointed out that there was considerable variation. Apparently, as the result of irritation, many antiseptics cause a flow of serous fluid. This is maximal with Dakin's solution. When 5 per cent. salt solution is applied to the wound there is also a considerable flow of serous fluid; the fluid is said in this case not to be due to irritation but to some other physical cause. The strong salt-solution, though probably not antiseptic, has a similar deleterious effect on emigration of leucocytes to that of an antiseptic.

As to the ultimate effect of antiseptic chemicals upon an infected wound-an effect which must be computed as its direct antibacterial power, plus its effect in causing serum to flow, less its evil influence upon the emigration of leucocytes - Mr. Fleming made some ingenious experiments with a chronic septic wound which seemed to show that neither the instillation of, nor the irrigation with, an antiseptic has any great influence in diminishing the number of microbes in the wound.

We think that as the result of $\mathrm{Mr}$. Fleming's experiments it must be accepted that the majority of antiseptics as used nowadays in infected wounds exert little or no direct bactericidal action. From this general statement we would make important exception in the case of the hypochlorite and the flavine group of antiseptics. The reasons for excepting these are as follows: In the case of the hypochlorite group Parry Morgan and others have shown that they act very rapidly, and before the solution has time to lose efficacy by dilution, and clinical experience with Dakin's solution and to a lesser extent with eusol seems to support the experimental evidence. (Fleming's experiment related below is an additional argument in favour of eusol.) With flavine and acriflavine we are dealing with chemicals which have an incomparably greater inhibitory and bactericidal effect in serum than any other drug; clinical results are at any rate not antagonistic, and we do not consider that Fleming's experiments were quite so convincing in the case of these drugs. But in the case of carbolic acid, perchloride of mercury, solution of tinct. iodi, boracic acid, and the many other antiseptics not specially excepted above, we consider they do no more good but probably more harm in an infected wound than a sterile solution of normal saline. If, however, there is any doubt about the sterility of the fluid to be used, then an advantage is gained by adding a small proportion of an antiseptic to render it sterile.

Wounds made by a High-velocity Projectile -Gunshot Wounds. - If a bullet traverses the body without injuring any vital structure, and carries on it or with it no infective material, then so long as the wound is protected from infection by a sterile dressing healing takes place without any serious con. sequences. If, however, bacteria are carried in by the projectile, then that wound must be regarded as infected from the start, since the momentum causes the microbes to be distributed widely in the tissues so that no antiseptic can reaoh them. The injured tissues which, in the absence of infection, would undergo aseptic necrosis and absorption, provide suitable and ready pabulum for the multiplying bacteria and, drainage being bad, infection spreads rapidly.

For such a primarily deeply-infected wound no ordinary antiseptic can be of any avail, for the microbes are inaccessible. The insertion of pastes and powders seems equally useless, qua antiseptics. At the same time the beneficial effects which have sometimes 
followed the application of antiseptics to a gunshot wound may be explained in another way. Wright and Fleming show that many antiseptics by their irritant action produce a flow of serum which may have an antibacterial action, whilst in the case of iodoform (and presumably B.I.P.P.) a beneficial action is generally acknowledged, though the reason for it is unknown. Concerning iodoform, Lister wrote :-

"Iodoform is a very peculiar antiseptic having extremely little influence over the growth of bacteria outside the body. But though such is the case, it is nevertheless unquestionably true that iodoform exercises a powerful antiseptic influence upon wounds!"

Lister favoured Behring's suggestion that iodoform produces its beneficial effects, not by acting directly upon the bacteria but by inducing chemical changes in the toxic products.

With regard to B.I.P.P., Fleming shows that it has no repellent action on leucocytes, and suggests that its beneficial action is largely due to its allowing free play to the activity of the leucocytes.

Though we have put in this evidence in favour of B.I.PP. and iodoform, we do not recommend their use save as a last resort. There is no doubt that when possible the best treatment of the class of wound we are discussing is by excising the infected track and draining; or if complete excision is impossible, to excise as much as possible and regard as a contaminated wound.

The fifth class is that of the granulating wound. Here the natural tissue-defence has been marshalled against the invading host of germs. The wonderful supply of bloodvessels in granulation tissue constitutes an elaborate line of communications for the supply of leucocytes and serum to the surface of the wound where the combat with the bacteria is waging. The flow of laudable pus is a good sign in a badlyinfected wound, since it signifies wellestablished granulation-tissue. In wounds which have reached this stage it is importa1 not to damage the granulations. They may be damaged by accumulated discharges $\frac{\vec{m}}{\mathrm{~m}}$ therefore adequate drainage is essentiad. They may be injured by irritating foreigy bodies, such as cloth or metal fragments $\frac{\bar{s}}{\overrightarrow{0}}$ therefore such should be removed whem practicable. They may be detrimentally affected by strong antiseptics-therefore such chemicals should never be applied $\vec{\oplus}$ healthy granulations. How, then, shou售 the organisms in a granulating wound destroyed. As a rule, if drainage be good and no foreign bodies present, healirig gradually takes place. The removal of the discharges may be facilitated by washing out the wound with normal saline solution. If the sterility of the fluid is not assuref, there is some advantage in adding a sma l quantity of an antiseptic to the solutio merely to make sure that the solution $\mathbb{P}_{\mathrm{TS}}$ sterile. Most antiseptics have little oro effect in reducing the number of organismb on the surface of a granulating wound. experiments by Parry Morgan on films of pus in vitro, and investigations by Fleming on granulating wounds in vivo, show that the hypochlorous solutions are likely to have $\overrightarrow{0}$ definite bactericidal effect on the organisnas on the surface of a granulating wound 高 used merely as a lotion to wash the surface. Parry Morgan's work shows that eithegr. Dakin's solution or eusol in strength I on 200 available chlorine will kill the bacteri in a film of pus within one and a ha्g్f minutes. Mr. Fleming's experiment is follows. He writes :-

"There is only one way in which? I have been able to sterilize the surface of a granulating wound by means of a chemical antiseptic. Such a wou was taken and divided into two halve्gुs. One half was washed with nornal saline and the other with eusol; i i pression cultures were then made, and it was found that there was no vefy great difference between the two halve्s. The whole wound was then washథ్ఠ d 
with eusol, and cultures were again made from the two halves, when it was found that the part which had been originally washed with saline was sterile, while the half which had been washed twice with eusol still gave a growth. A preliminary wash with carbolic acid before the eusol did not enable the latter to sterilize the surface. It would seem that the saline solution removed the albuminous material from the surface of the wound, and then the eusol was able to act on the bacteria in practically a watery medium, in which it is very active; this would seem to be the best way of ridding the surface of a wound of bacteria."

The very valuable suggestion has been made by Parry Morgan that all antiseptics (except those actually incompatible with salt) should be made up with normal saline instead of plain water. $\mathrm{He}$ points out that plain water or any hypotonic solution is likely to make the cells on the surface of the wound swell up and so prevent the salutary flow of serum.

Though we have in the foregoing come to the conclusion that the majority of antiseptics do very little, if any, good in the case of a deeply-infected or granulating wound by any bactericidal power they possess, there is no doubt that they may be of some benefit in three ways. . In the first place the irrigation does good by clearing away debris; secondly, the irritant action of some antiseptics causes a flow of serous fluid which has antibacterial properties; thirdly, some chemicals have a solvent effect on sloughs. In any case an antiseptic solution will be sterile and not introduce any other organism into the wound.

The comparative failure of antiseptics to purify infected wounds during the war led to researches having as their object the reinforcement of the natural resistance against infection. No substance was found which could greatly increase the emigration of leucocytes, but several chemicals were found to increase the exudation of serous fluid into the wound. Sir Almroth Wright found that, following the application of 5 per cent. solution of common salt, a free flow of serous fluid took place from the wound, and offered the opinion that probably most of the benefit of any antiseptic applied to the wound was owing to the irritative flow of serum thereby occasioned. Following this, Wright devised a simple yet ingenious technique for treating wounds by a physiological method. This method is apparently applicable to a wound in any stage. The aim is to get a plentiful supply of both serum and leucocytes in the wound. It is impossible to gain both these objectives by the use of hypertonic saline, for that solution is one which repels leucocytes and banishes them from the field. Therefore an application of the 5 per cent. saline is followed by a dressing with normal saline, for the latter permits, or even encourages, the normal course of emigration of the white cells. The stronger salt-solution is very inimical to leucocytes, and causes some to disintegrate; by this means some tryptic ferment is liberated which helps to digest sloughs in the wound, and aids in cleaning it up. Though there are some theoretical objections against the salt treatment, it appears to work fairly satisfactorily, and may be regarded as a useful alternative method in the treatment of wounds with established infection, in which antiseptics have but a limited effect. But we think it unwise to use it for superficially contaminated wounds in which experimental evidence favours the use of antiseptics, and of course, in every possible case, partial or complete excision of the wound should be carried out as a preliminary.

An extension of this treatment was to put packs of solid salt into the wound. This method has all the disadvantages of the insertion of an irritating foreign body and none of the advantages of an antiseptic, and the published results of this treatment were not encouraging to the impartial observer.

Before summarizing the foregoing, we 
must consider the effect of antiseptics on surfaces which are unbroken. In the case of skin there is little doubt that microbes on the surface may be destroyed by certain antiseptics. The simpler the technique the better. Probably the application of tincture of iodine or saturated solution of picric acid is as good as any, though many elaborate techniques have been published. It is very doubtful if any antiseptic can kill microbes in the depth of the skin without damaging the skin itself. Our personal experience of gangrene of the fingers (after the application of carbolic acid by other people) never inclined us to use carbolic in the preparation of the skin, but in view of the many accidents involving hairy parts which are often begrimed with dirt which cannot easily be removed, we are impelled to quote Lord Lister's advice as to carbolic. He wrote :-

"So also with the purification of the skin of the patient. It is not needful to apply an antiseptic lotion for hours together, as is sometimes done; a few minutes' action of the $I$ in 20 carbolic solution is really sufficient; while its long-continued application sometimes produces troublesome irritation. . . . For my own part I do not even use soap and water. I trust to the carbolic acid which, by its penetrating power and great affinity for organic substances, purifies the integument as corrosive sublimate cannot."

The experiment whereby Lister demonstrated the avidity of carbolic acid for the epidermic tissues was very striking. "Having discovered a method by which the amount of carbolic acid present in a watery solution could be determined, he packed a test tube closely with hair of the human head and added just enough 5 per cent solution of carbolic acid to cover it, eight times the weight of the hair being required for the purpose. Half an hour later he poured out some of the liquid and applied the test, when it was found that already nearly half the carbolic acid had been withdrawn by the hair from the watery solution."

Considering that the hair was only a eighth part of the weight of the solution this was certainly very remarkable.

The rarity of suppuration after operatio on cases of acute abdominal disease shows at any rate, that the old method of antiseptic. compress was unnecessary and can be dis: pensed with.

Should antiseptics be applied to mucous surfaces? If there is an intact mucosa? there is little chance of any antiseptic reachis ing the depth of the mucous crypts wheros the microbes lodge, and certainly the delicate surface of the membrane can easilis be injured by chemicals. The main purpos $\vec{E}$ of any lotion used to a mucous membrane is to cleanse the discharge from the surface of the mucosa. For this purpose saline quite suitable. Very dilute antiseptics are permissible, for the antiseptic ensures steritity of the lotion. We think all recent experdio mental work is against the use of strong antiseptics for mucous membranes. Clinica experience asserts that benefits follow the use of antiseptic lotions, but such results are not so readily controlled as in the cas $\overrightarrow{\vec{E}}$ of badly contaminated wounds. To discuss this question would lead us too far awat from wounds, but we think that the use of perchloride of mercury and similar anti septics in such mucous cavities as the bladder and uterus is probably as oftem harmful as beneficial.

To summarize the foregoing :-

In clean wounds-those known not to be contaminated-there is no need fo․ㅡㄹ. antiseptics.

In superficially contaminated woundsi which are seen within a few hours of in fliction, primary sterilization of the wounce should be aimed at by use of antiseptics.

In wounds with well-established infection most antiseptics do little good beyond washing, and many do much harm by. injuring the tissues. However, the hypo: chlorite solutions and flavine derivatives, used $\mathbb{R}$ 
with a full knowledge that they are of limited value, may be tried.

Another method for wounds of this nature is the alternative application of hypertonic and normal saline, according to the technique recommended by Sir Almroth Wright.

Granulating wounds are best treated by washing out with normal saline, or saline and eusol (see text). There is no need to wash out an ordinary abscess cavity.

Antiseptics can be applied to skin for sterilization purposes, but it is probably never wise to apply them to mucous surfaces except in very dilute solutions to wash the surface.

There is no doubt antiseptics have often been used perfunctorily and irrationally, and a great deal too much has been expected of and claimed for them. As Parry Morgan, a very judicial observer, states :-

"Antiseptics cannot do as much as is claimed for them. They certainly cannot sterilize the tissues subjacent to the surface of a wound, and, indeed, cannot be depended upon to sterilize an accessible surface, although they kill many of the organisms on it. . . . If we expect more from the antiseptics at present available we shall be disappointed."

The importance of realizing this, as Fleming wisely points out, is that if the surgeon once realizes that an antiseptic is but an accessory in the technique of wound treatment, he will be much more careful in the other parts of the treatment, i.e., the excision of damaged tissues, the provision of adequate drainage, and the careful dressing so that no other organisms get into the wound. Fleming, from an examination of wounds observed at a base hospital during the war, came to the conclusion that faulty technique in dressing the wounds was responsible for the almost universal presence of the streptococcus-the most dangerous of all the microbes found in wounds. $\mathrm{He}$ therefore pleads for a more lavish use of chemical antiseptics outside the wound. All the things which come near a wound should be sterilized by heat or antiseptics, and in the case of every raw surface a dressing should be applied which should not only be itself sterile and cover considerably more than the area of the wound, but it should also contain some substance which should at least inhibit the growth of organisms in the serous discharges which soak the dressings, and kill any stray organisms which contaminate the outer part of the dressing. But it must be remembered that the presence of an antiseptic in the dressing should not in any degree cause the surgeon to abate his meticulous care in dealing with the wound.

\section{MEDICINE AND THE STATE.}

SUMMARY OF A LECTURE DELIVERED

$$
\begin{gathered}
B y \text { DAVID C. KIRKHOPE, } \\
\text { M.D., cH.M., D.P.H., }
\end{gathered}
$$

Medical Officer of Health; Medical Officer, Tottenham Maternity and Child Welfare Committee.

AT THE PRINCE OF WALES hospital, TOtTENham, ON JUNE 28, 1927.

IT would be impossible in the time at my disposal to give anything like a complete account of the relations of the State with Medicine. Yet it appears to be necessary, or at least desirable, that at one time or another I should treat of the subject, at any rate, broadly. The Ministry of Health was formed to consolidate all the health services in their central administration within itself. This has not yet been accomplished. For the Board of Education -in respect of children of school age-the Home Office in respect of prisons and factories (and? lunatic asylums), and the Ministry of War in respect of the Army and Navy, have their own special medical departments. I do not purpose to deal with all of these, and in respect of the Ministry of Health and Board of Education only in so far as their oversight concerns the adminis- 